\title{
Study on the Market Risk Measurement of the Style Portfolios in Stock Markets Based on EVT-t-Copula Model
}

\author{
http://dx.doi.org/10.3991/ijoe.v9iS2.2595 \\ Y.H. Zhou ${ }^{1}$ and W.W. Guo ${ }^{2}$ \\ ${ }^{1}$ South China University of Technology, Guangzhou, China \\ ${ }^{2}$ Guangdong University of Business Studies, Guangzhou, China
}

\begin{abstract}
For the presence of non-normal distribution characteristics in the financial assets returns, the model of $\operatorname{AR}(1)-G J R(1,1)$ is used to characterize the marginal distribution of the style assets in China stock market. The Copula function is introduced to analyze the dependency structure between the six style assets, combined with the marginal distributed residual sequences. And the joint return distribution of the style portfolios is simulated, combined with extreme value theory and Monte Carlo simulation method. Then the market risks (VaR and CVaR) of the style portfolios in China stock markets are obtained. The results of the study show that the generalized Pareto distribution Model can well fit the non-normal distribution characteristics such as peak and fat tail in the style assets returns.
\end{abstract}

Index Terms-Style assets in stock markets, Market risk, Extreme Value Theory, Multivariate Coupla Model

\section{INTRODUCTION}

In 1952, Markowitz presented the mean-variance model for portfolios [1]. Markowitz's mean-variance model adopts Pearson's linear correlation coefficient to reflect the correlation among the benefits of financial capitals. Since Pearson's linear correlation can't describe nonelliptical distribution and can't measure the correlation among random variables according to the joint distribution of random variables. These defects result in its obvious errors when describing the nonlinear dependency features among various style assets and measuring risk. Since Copula function has overcome the shortcomings of traditional risk theory, it can be used to connect the marginal distribution and joint distribution of random variable. In addition, the marginal distribution is not required to have the same distribution form. Then structure the flexible and practical multivariate distribution to describe the true distribution and related structures of the yields on financial assets. Because the evaluation of the model is simple, the economic meaning is obvious and the Non-linear, non-symmetrical and the tail correlation can be captured when Copula function is used to structure multivariate financial model. Consequently, as the non-linear correlation model that is closer to reality, there has been more and more studies and applications of the risk measurement model based on Copula function in the field of risk management for portfolios over the years.

\section{LITERATURE REVIEW}

Sklar(1959) first proposed that a joint distribution can disintegrate into $\mathrm{k}$ marginal distribution and one Copula function. The Copula function describes the dependency structures among variables[2].The overseas practical application of Copula function in financial terms began in 1999. Bouye.E, etc. (2000) made systematic introduction about some financial applications of Copula[3]. Copula technology is modeling the entire joint distribution and it can be easily extended to the case of conditional distribution. In empirical studies, Claudio Romano (2002) applied Copula theory and extreme value theory to research the risk of portfolios in Italy capital market[4]; Rosenberg and Schuermann(2006) used Copula theory to carry out comprehensive research on the aggregation problems of market risks, credit risks and operational risks[5].

At home, Zhang Yaoting(2002) explored the practical feasibility of Copula in financial field theoretically[6]. Liu Qiongfang (2010) [7], Hu Xinhan (2011) [8], Yi Wende (2012) [9], etc. made correlational researches about the applications of Copula function in financial field as well. All in all, the domestic financial risk analysis based on the binary Copula function has been increasingly rich so far. But the major research results remain basically in the measurement level of single or two-dimensional portfolio risks. Obviously, there is a large gap between investment practices and this. At the same time, the characteristics between various assets such as correlation, nonlinear, etc. have not been fully considered in the risk measurement. The ignorance of the inherent characteristics of financial assets will have an immeasurable influence on the risk assessment [10]. Thus the Copula function will be introduced to analyze the dependency structure between multiple style assets in China stock market. And simulate the joint return distribution of the style portfolios in China stock market, combined with the extreme value theory and the Monte Carlo simulation method. Finally, the market risk (VaR and CVaR) of the style portfolios in China stock markets is obtained.

In this article, our research objective is the market risk measurement of the style portfolios in China's stock market (large growth, large value, mid-cap growth, midcap value, small cap growth, small cap value). As the return series of the style assets in the present stock market show the distribution characteristics such as "self-related", "spike", "fat-tailed", "biased", "volatility" and so on. The 
marginal distribution model of the return series of the style assets is established by adopting the model of AR(1)$\operatorname{GJR}(1,1)$. Strive to capture the sundry non-normal distribution characters shown by the return distribution of the style assets. Next, the t-Copula function is selected to connect the marginal distributions. The joint distribution function of the returns of the six style assets is established. In addition, simulate the joint return distribution of the style portfolios in China stock markets combined with the extreme value theory and the Monte Carlo simulation method. Finally, the market risk (VaR and CVaR)of the style portfolio in China stock markets is obtained. The results of the study show that the generalized Pareto distribution based on the extreme value theory can well fit the tail behavior of the daily return series of the style assets. The remaining structure of the article: The second part is the literature review, the third part is the model, the fourth part is the data description, the fifth part is the empirical results and the final part is the conclusion.

\section{MODEL}

\section{A. The Model of AR(1)-GJR(1,1)}

The return series of financial assets show the characteristics such as "autoregression", "conditional heteroscedasticity", "biased", "fat-tailed" and "spike". Meanwhile, the aggregation and asymmetry of the fluctuation of the return series are taken into account. The model of $\operatorname{AR}(1)-G J R(1,1)$ is used to characterize the distribution characteristics of the returns of the related financial assets:

$$
\begin{gathered}
R_{i, t}=c_{0}+c_{1} R_{i, t-1}+e_{i, t} ; \mathrm{i}=1,2, \mathrm{~L} 6 \\
e_{i, t}=h_{i, t} \varepsilon_{i, t}, \varepsilon_{i, t}: \operatorname{SkT}(v, \lambda) \\
h_{i, t}^{2}=\omega_{i, t}+\alpha e_{i, t-1}^{2}+\beta h^{2}{ }_{i, t-1}+\gamma e_{i, t-1}^{2} I\left(e_{i, t-1}<0\right)
\end{gathered}
$$

Apparently, there are 8 parameters in each marginal distribution model. Among them, formula(1)is a boundary equation, containing parameter $\mathrm{C}_{0}$ and $\mathrm{C}_{1} \cdot \mathrm{e}_{\mathrm{i}, \mathrm{t}}$ is the residual of the return of each style asset. $i=1,2, \mathrm{~L} 6$ indicate large growth, large value, mid-cap growth, midcap value, small-cap growth and small-cap value style assets respectively; Formula(2)is a skewness distribution function, containing parameter $\mathrm{v}$ and $\lambda$; Formula (3) is a variance equation, including four parameters $(\omega, \alpha, \beta, \gamma) . \mathrm{I}\left(e_{i, t-1}<0\right)$ is an indicative index, when $e_{i, t-1}<0$ take 1 , otherwise 0 . It shows that the fluctuation of the return series when facing a negative impact is larger than the one when facing a positive impact.

For the model of $\operatorname{GJR}(1,1)$, formula (3) is also facing the following constraint conditions:

$$
\alpha+2 \beta+\gamma<2, \alpha>-\gamma, \beta \in(0,1)
$$

\section{B. The POT Extreme Value Theory Model}

The POT model is adopted to estimate relevant parameters. The distribution of random variable $\mathrm{X}$ when it surpasses some threshold $F_{u}$, should be concerned in POT model. Among them, $\mathrm{F}$ is the distribution function of $\mathrm{X}$. In general, the distribution function $F_{u}$ is called the extreme conditional loss distribution function. In practice, the historical simulation method is usually used to do the estimation.
$F(u)=\left(n-N_{u}\right) / n, \mathrm{n}$ is the sample size, $N_{u}$ is the observed quantity that exceeds the threshold. Plug $F(u)$ into the above formula and the tail estimation can be obtained:

$$
\hat{F(x)}=\left\{\begin{array}{c}
1-\frac{N_{u}}{n}\left(1+\frac{\xi}{\sigma}(\mathrm{z}-u)\right)^{1 / \xi}, \xi \neq 0 \\
1-\frac{N_{u}}{n}\left(e^{-(\mathrm{z}-\mathrm{u}) / \sigma}\right), \xi=0
\end{array}\right\}
$$

The value of the shape parameters $\xi$ is proportional to the tail thickness of the return series of the style assets. The bigger $\xi$ is, the thicker the tail of the return series will be. When the return series of financial assets directly uses EVT, the estimated $\xi$ must be greater than zero due to the spike and fat tail of the series. Nonetheless, the obtained $\xi$ is not necessarily required to be greater than zero due to the extreme value analysis of the residual sequence in our model. The logarithmic likelihood method can be used to estimate the extreme value parameters

\section{The Disintegration and Construction of the Multivariate Copula Model}

Sklar(1959)first proposed that a joint distribution can disintegrate into $\mathrm{k}$ marginal distribution and one Copula function. The Copula function described the correlation between the variables. Let $F(\mathrm{~g}, \mathrm{~L}, \mathrm{~g})$ be the joint distribution function that have the marginal distribution $\mathrm{F}_{1}(\mathrm{~g}), \mathrm{F}_{2}(\mathrm{~g}), \mathrm{L}, \mathrm{F}_{\mathrm{N}}(\mathrm{g})$. So there is a function $\mathrm{C}(\mathrm{g}, \mathrm{L}, \mathrm{g})$ conforming to the following formulas.

$$
F\left(x_{1}, \mathrm{x}_{2}, \mathrm{~L} \quad x_{N}\right)=\mathrm{C}\left(\mathrm{F}_{1}\left(x_{1}\right), \mathrm{F}\left(\mathrm{x}_{2}\right), \mathrm{L}, \mathrm{F}_{\mathrm{N}}\left(x_{N}\right)\right)
$$

For the research problem in the paper, we use $R_{i}(i=1,2, \mathrm{~L} 6)$ to express the return series of the six kinds of style assets such as large growth (LG), large value (LV), mid-cap growth (MG), mid-cap value (MV), small-cap growth (SG) and small-cap value (SV) respectively. Let the joint distribution function and the probability density function of $\left(R_{1}, \mathrm{~L} R_{6}\right)$ be $\mathrm{F}$ and $f$ respectively, so the disintegration can proceed as follows:

$$
f\left(R_{6} \mid R_{1}, \cdots, R_{5}\right)=\frac{f\left(R_{5}, R_{6} \mid R_{1}, R_{2}, R_{3}, R_{4}\right)}{f\left(R_{5} \mid R_{1}, R_{2}, R_{3}, R_{4}\right)}=c_{5,6 \mid 1,2,3,4} \times f\left(R_{5} \mid R_{1}, R_{2}, R_{3}, R_{4}\right)
$$

Where, $c(\cdot)$ is the conditional Copula density function. For simplicity, with regard to random subscripts $i, j, \quad i<j$ and $i_{i}<\cdots<j_{k}$ are given. So: ${ }^{c_{i, j} \mid i_{1}, l_{k}}=c_{i, j i_{1, L}, l_{k}}\left(F\left(R_{i} \mid R_{1_{1}}, \mathrm{~L} \quad, R_{i_{1}}\right) F\left(R_{j} \mid R_{i_{1}}, \mathrm{~L} \quad, R_{i_{1}}\right)\right)$.

\section{DATA DESCRIPTION}

The style index series (large value, large growth, midcap value, mid-cap growth, small-cap value and small-cap growth) launched by CITIC S\&P is adopted in this article.All the sample data is the rehabilitated daily index closing quotation. The period of the research is from February 27, 2004 to September 11, 2012. There are 2081 sample data as a whole. The index returns of the relevant style assets are adopted to represent the market return of this type of style asset. Every series has 2080 return data and they are given by:

$$
R_{i, t}=100 \times \operatorname{Ln}\left(P_{i, t} / P_{i, t-1}\right)
$$

Where ${ }^{R_{i, t}}$ represents the logarithmic return of index $i$ during the No. ${ }^{t}$ period; ${ }^{P_{i, t}}$ represents the closing price of 
index $i$ at the end of No. $t$ period; ${ }^{P_{i, t-1}}$ represents the closing price of index $i$ at the end of No. ${ }^{t-1}$ period. $R_{i}(i=1,2, \mathrm{~L} 6)$ represents the return series of the six kinds of style assets such as large growth (LG), large value (LV), mid-cap growth (MG), mid-cap value (MV), smallcap growth (SG) and small-cap value (SV) respectively. The descriptive statistics of the return series of each style asset are given in Table I. From Table I we can learn that all the returns of the style indexes show the characteristics such as left-skewed and spike. Seen from the JB test, all the returns of the style indexes reject the hypothesis of normal distribution in $1 \%$ confidence level. The stationary test is launched on the sample data. Seen from the ADF statistics and $\mathrm{P}$ value, all of the return series of the style indexes reject the null hypothesis in the $99 \%$ significance level. This goes to show that all of the return series of the style indexes are left-skewed, peaky and stationary time series.

\section{EMPIRICAL RESULTS}

(1) The estimation results of the AR(1)GJR(1,1)marginal distribution function.

As the return series of the style asset index shows the characteristics such as self-related, time-varying, biased, volatility, spike, fat-tailed and so on, $\operatorname{AR}(1)-G J R(1,1)$ is used to conduct the description in this article. Thus the marginal distribution function of each style assets series can be structured. The estimation is conducted by the Matlab2011b software programming. Then the marginal distribution parameters of the return series of each style asset are obtained. The specific results are shown in Table II. The K-S test method is used to examine whether the transformed series obeys the(0,1)uniform distribution or not. Both the K-S statistics and their probability values in Table II show that: Both the two series accept the null hypothesis that "the transformed series obeys the( 0,1$)$ uniform distribution". The result also shows that there is no autocorrelation in each transformed series. Consequently, we can assume that the two series are independent after the transform. All these show that the model of AR(1)-GJR(1,1)can fit well with the conditional marginal distribution of each series.

The conditional distribution of the return series of each style asset can be confirmed according to the model of AR(1)-GJR(1,1)acquired from the estimation. The two new series $\left\{u_{t}\right\}$ and $\left\{v_{t}\right\}$ that obey the $(0,1)$ uniform distribution can be acquired after the probability integral transform of the primitive series, based on the obtained conditional distribution. Then we can get ready for the latter relevant empirical analysis.

TABLE I. HE DESCRIPTIVE STATISTICS OF THE RETURN SERIES OF THE STYLE ASSETS(20040301-20120911)

\begin{tabular}{|c|c|c|c|c|c|c|c|c|c|}
\hline Style assets & Mean value & Median & Maximum value & Minimum value & Standard deviation & Skewness & Kurtosis & Jarque-Bera & Probability \\
\hline LG & 0.0417 & 0.0532 & 9.2336 & -9.7917 & 1.9608 & -0.2256 & 5.4052 & 518.9979 & 0.0000 \\
\hline LV & 0.0294 & 0.0547 & 9.2738 & -9.4277 & 1.9266 & -0.3979 & 6.0224 & 846.5529 & 0.0000 \\
\hline MG & 0.0369 & 0.0997 & 9.2582 & -9.8924 & 2.0710 & -0.4162 & 5.1320 & 453.9613 & 0.0000 \\
\hline MV & 0.0429 & 0.1426 & 9.1857 & -9.6094 & 2.0374 & -0.5330 & 5.5711 & 671.4072 & 0.0000 \\
\hline SG & 0.0534 & 0.1436 & 9.3494 & -9.0975 & 2.1192 & -0.5081 & 5.4579 & 613.0864 & 0.0000 \\
\hline SV & 0.0468 & 0.1977 & 9.3416 & -9.2657 & 2.1244 & -0.6820 & 5.6166 & 754.5818 & 0.0000 \\
\hline
\end{tabular}

Date Source: Juyuan database

TABLE II. THE ESTIMATION RESULTS OF THE MARGINAL DISTRIBUTION PARAMETERS OF THE RETURN SERIES OF THE STYLE ASSET INDEXES

\begin{tabular}{|c|c|c|c|c|c|c|c|c|c|c|c|}
\hline & $C_{0}$ & $C_{1}$ & $\omega$ & $\alpha$ & $\beta$ & $\gamma$ & $v$ & $\lambda$ & $\begin{array}{c}\text { Log } \\
\text { likelihood } \\
\end{array}$ & $\begin{array}{c}\text { K-S } \\
\text { statistics } \\
\end{array}$ & $\begin{array}{c}\text { K-S probability } \\
\text { value }\end{array}$ \\
\hline \multirow{2}{*}{ LG } & 0.02 & 0.03 & $0.03 * *$ & $0.05 * * *$ & $0.94 * * *$ & 0.01 & $7.22 * * *$ & -0.03 & \multirow{2}{*}{-4104.39} & \multirow{2}{*}{0.015} & \multirow{2}{*}{0.67} \\
\hline & $(0.06)$ & $(0.022)$ & $(0.014)$ & $(0.011)$ & $(0.014)$ & $(0.014)$ & $(1.1)$ & $(0.021)$ & & & \\
\hline \multirow{2}{*}{$\mathbf{L V}$} & -0.01 & 0.03 & $0.02 * *$ & $0.06^{* *}$ & $0.94 * *$ & 0.00 & $6.39 * *$ & $-0.05 * *$ & \multirow{2}{*}{-4016.92} & \multirow{2}{*}{0.017} & \multirow{2}{*}{0.65} \\
\hline & $(0.053)$ & $(0.022)$ & $(0.018)$ & $(0.015)$ & $(0.026)$ & $(0.03)$ & $(0.886)$ & $(0.028)$ & & & \\
\hline \multirow{2}{*}{ MG } & -0.01 & $0.07 * *$ & $0.06^{* * * *}$ & $0.06 * * *$ & $0.92 * * *$ & 0.02 & $8.48 * * *$ & $-0.14 * * *$ & \multirow{2}{*}{-4226.02} & \multirow{2}{*}{0.013} & \multirow{2}{*}{0.72} \\
\hline & $(0.041)$ & $(0.023)$ & $(0.023)$ & $(0.014)$ & $(0.017)$ & $(0.016)$ & $(1.501)$ & $(0.032)$ & & & \\
\hline \multirow{2}{*}{ MV } & -0.01 & 0.03 & $0.04 * *$ & $0.07 * * *$ & $0.92 * * *$ & 0.00 & $6.97 * * *$ & $-0.18 * * *$ & \multirow{2}{*}{-4150.41} & \multirow{2}{*}{0.012} & \multirow{2}{*}{0.75} \\
\hline & $(0.014)$ & $(0.03)$ & $(0.024)$ & $(0.014)$ & $(0.022)$ & $(0.019)$ & $(1.08)$ & $(0.026)$ & & & \\
\hline \multirow{2}{*}{ SG } & 0.02 & $0.05^{* *}$ & $0.09 * * *$ & $0.07 * * *$ & $0.90 * * *$ & 0.03 & $8.40 * * *$ & $-0.19 * * *$ & \multirow{2}{*}{-4243.70} & \multirow{2}{*}{0.014} & \multirow{2}{*}{0.69} \\
\hline & $(0.041)$ & $(0.021)$ & $(0.031)$ & $(0.014)$ & $(0.02)$ & $(0.019)$ & $(1.468)$ & $(0.03)$ & & & \\
\hline \multirow{2}{*}{ SV } & 0.01 & 0.02 & $0.07 * *$ & $0.08 * * *$ & $0.91 * * *$ & 0.00 & $7.03 * * *$ & $-0.26 * * *$ & \multirow{2}{*}{-4227.98} & \multirow{2}{*}{0.015} & \multirow{2}{*}{0.68} \\
\hline & $(0.111)$ & $(0.027)$ & $(0.039)$ & $(0.017)$ & $(0.026)$ & $(0.033)$ & $(1.078)$ & $(0.046)$ & & & \\
\hline
\end{tabular}

Note: 1、Standard errors in parentheses $2, * * * \mathrm{p}<0.01, * * \mathrm{p}<0.05, * \mathrm{p}<0.1$

(2) The empirical cumulative distribution function of the return series of each style portfolio based on extreme value theory.The independent identically distributed residual sequence with standardization $\varepsilon_{i, t}=e_{i, t} / h_{i, t}$ can be acquired according to formula (2). After that, the Gaussian kernel estimation can be used to acquire the empirical cumulative distribution function of the return series of each style asset. The method can eliminate the ladder-like structure caused by illbehaved samples of the estimation of general cumulative distribution function. But the effect is not good when the method of kernel estimation is applied to the upper and lower tail estimation of cumulative distribution function. In this 
regard, we use extreme value theory to estimate the distribution of the tail part, in order to acquire better effects of the estimation[11].

For simplicity, we only estimate the lower-tail risk part of the equally-weighted return series of the style portfolios based on POT model. The specific result is shown in Table III. Table III is the graph of the cumulative distribution of the one-month simulated returns of the six style portfolios based on the multivariate t-Copula model. We could see that the graph of the cumulative distribution based on the multivariate $\mathrm{t}$ Copula model is less affected by extreme values.

Now the multivariate $\mathrm{t}$-Copula model is used to conduct the next-month simulation in the future (The trading days are assumed as 22 days.). The daily simulations are 2000 times. The residual sequence that obeys the $\mathrm{U}(0,1)$ uniform distribution is made. The residual sequence is disposed to get the newly independent identically distributed residual sequence that obeys standardization. Perform the GARCH simulation on the new residual sequence. The $22 \times 2000 \times 6$ simulation return series is generated. The style portfolio returns constituted by the simulation return series of the style assets are generated according to the equally weighted proportion (Namely each style asset has a one sixth interest). Afterwards, the $\mathrm{VaR}$ and $\mathrm{CVaR}$ values of the portfolio return series in the $95 \%$ and $99 \%$ confidence levels are obtained. The specific result is shown in Table IV.

TABLE III. THE LOWER-TAIL THRESHOLD VALUE AND THE ESTIMATION VALUE OF THE PARAMETERS BASED ON POT MODEL

\begin{tabular}{|c|c|c|c|c|c|}
\hline $\begin{array}{r}\text { Extreme } \\
\text { value } \\
\text { model }\end{array}$ & $\begin{array}{c}\text { ponfidence } \\
\text { level } \\
\mathbf{p}\end{array}$ & $\begin{array}{c}\text { Threshold } \\
\text { value } \\
u\end{array}$ & $\begin{array}{c}\text { Shape } \\
\text { Parameter } \\
\xi\end{array}$ & $\begin{array}{c}\text { Location } \\
\text { parameter } \\
\mu\end{array}$ & $\begin{array}{c}\text { Dimension } \\
\text { parameter } \\
\sigma\end{array}$ \\
\hline \multirow{2}{*}{ POT } & 0.95 & 3.22 & $-0.31 * * *$ & $-8.47 * * *$ & $6.00 * * *$ \\
\cline { 2 - 6 } & 0.99 & 6.43 & $-0.21 * *$ & $-2.16 * *$ & $2.94 * *$ \\
\hline
\end{tabular}

Note: $* * * \mathrm{p}<0.01, * * \mathrm{p}<0.05, * \mathrm{p}<0.1$

TABLE IV. THE COMPARISON BETWEEN VAR AND CVAR VALUE BASED ON EVT-T-COPULA MODEL SIMULATION AND EMPIRICAL DISTRIBUTION

\begin{tabular}{|c|c|c|c|c|c|c|}
\hline $\begin{array}{c}\text { Distribution } \\
\text { pattern }\end{array}$ & DOF & $\begin{array}{c}\text { Maximum } \\
\text { simulation } \\
\text { loss(\%) } \\
\end{array}$ & $\begin{array}{l}\text { Maximum } \\
\text { simulation } \\
\text { income(\%) }\end{array}$ & Type of Confidence level & $\begin{array}{c}\text { Risk value } \\
\text { type }\end{array}$ & $\begin{array}{c}\text { simulated } \\
\text { result } \\
(\%) \\
\end{array}$ \\
\hline \multirow{4}{*}{$\begin{array}{l}\text { EVT-t- } \\
\text { Copula }\end{array}$} & \multirow{4}{*}{5.55} & \multirow{4}{*}{-39.50} & \multirow{4}{*}{30.03} & \multirow{2}{*}{$95 \%$ confidence level } & $\begin{array}{l}\text { VAR } \\
\end{array}$ & -17.86 \\
\hline & & & & & CVAR & -24.60 \\
\hline & & & & \multirow{2}{*}{$99 \%$ confidence level } & VAR & -30.20 \\
\hline & & & & & CVAR & -34.40 \\
\hline \multirow{4}{*}{$\begin{array}{c}\text { Empirical } \\
\text { distribution }\end{array}$} & & \multirow{4}{*}{-29.65} & \multirow{4}{*}{34.34} & \multirow{2}{*}{$95 \%$ confidence level } & VAR & -14.36 \\
\hline & & & & & CVAR & -20.32 \\
\hline & & & & \multirow{2}{*}{$99 \%$ confidence level } & VAR & -23.84 \\
\hline & & & & & CVAR & -26.31 \\
\hline
\end{tabular}

Seen from Table IV: In the three confidence levels $(1 \%$ and $5 \%$ ), all of the obtained one-month period risk values (VAR and CAVR) of the style portfolios based on the EVT-t-Copula model are larger than the corresponding values based on empirical distribution. And the simulated maximum loss value can cover the actual maximum loss value. These results show that the EVT-t-Copula model is suitable for the simulation of the lower-tail risk of the style portfolios and its simulated result is relatively cautious. Moreover, all the VAR values based on the EVT-t-Copula model are less than the CVaR values in the same confidence level. This is consistent with the definition of $\mathrm{CVaR}$. This shows that it is relatively reasonable to analyze the market risk of the style portfolios in China stock markets based on extreme value theory and the t-Copula simulation technique.

\section{CONCLUSION}

We apply extreme value theory and the multivariate tCopula model to the risk value measurement of style portfolios in China stock markets in this article. Firstly, the $\operatorname{AR}(1)-\operatorname{GJR}(1,1)$ model is selected to characterize the six types of the return series of the style assets. Then the $\mathrm{t}$-Copula model is used to estimate the linearly dependent coefficient matrix and the corresponding degree of freedom respectively. The above linearly dependent coefficient matrix and degree of freedom are used to simulate the next-month period daily return series of the style assets. At the same time, the VaR and CVaR values of the style portfolios in different confidence level are obtained in the simulated return series. The results of the study show that the generalized Pareto distribution based on extreme value theory can well fit the lower-tail risk features of the return series of the style assets; The simulated results show that the EVT-t-Copula model can well characterize the market risks of the style portfolios in China stock markets.

\section{REFERENCES}

[1] Harry M.Markowitz, “ Portfolio Selection”, Journal of Finance, Vol.7, No.1, pp. 77-91,1952.

[2] A. Skar, "Fonctionde repartition a dimension stleurs marges", Publ, Inst, stat, Univ, Paris, Vol.8, pp. 229-231,1959.

[3] Bouyé, Eric, Durrleman, Valdo, Nikeghbali, Ashkan, Riboulet, Gaël and Roncalli, Thierry, Copulas for Finance - A Reading Guide and Some Applications (March 7, 2000). Available at SSRN: http://ssrn.com/abstract $=1032533$

[4] Claudio Romano, "Calibrating and Simulating Copula Functions: an Application to the Italian Stock Market", working paper, 2002.

[5] V. Joshua. Rosenberg and Til Schuermann., "A general approach to integrated risk management with skewed, fat-tailed risks", Journal of Financial Economics, Vol.79, No.3, pp. 569-614. 2006. http://dx.doi.org/10.1016/j.jfineco.2005.03.001

[6] Yaoting Zhang, "Copula technology and financial risk analysis", Statistical research, Vol.4, pp. 48-51, 2002.

[7] Qiongfang Liu, "Dependency research on financial time series based on the Copula theory",Ph.D. thesis,Chongqing university,Chongqing, China, 2010.

[8] Xinhan Hu, "Applications of the Copula method in portfolios and the risk measurement in financial markets", Ph.D. thesis , University of Science and Technology of China, Hefei, China,2011 
[9] Wende Yi, "Dependency model and its applications based on high order moment fluctuation and Copula function", Management review,Vol.24, No.1, pp. 58-66, 2012.

[10] Nikolai Kolev, Ulisses dos Anjos, Beatriz Vaz de M. , "Copulas: A Review and Recent Developments", Stochastic Models, Vol.22, No.4, pp. 617-660, 2006. http://dx.doi.org/10.1080/153 26340600878206

[11] Baisheng Cui and Langnan Chen, "Risk measurement of Chinese foreign exchange reserve rate based on extreme value theory and multivariate timevariant Copula model", Journal of International Trade,Vol.12, pp. 158-168, 2011.

\section{AUTHORS}

Y. H. Zhou is a $\mathrm{PhD}$ candidate at School of Business Administration and a lecturer in the School of Economics and Commerce in South China University of Technology, Guangzhou, 510641 China. Her research interests include financial management and capital operation, etc. (e-mail: yhzhou2013@163.com).

W.W.Guo graduated from South China University of Technology and is a lecturer in the Finance Department at Guangdong University of Business Studies, Guangzhou, 510640. P.R.China. His research interests include fund investment and financial credit risk analysis, etc (e-mail: yhzhou2013@163.com).

This work was supported in part by: National Youth Foundation for Social Science (12CJY006), Guangdong Foundation for Natural Science (S2012040008073), Guangdong Soft-science Research Foundation (2012B070400004), Foundation for Distinguished Young Talents in Higher Education of Guangdong, China (2012WYM_0068). This article is an extended and modified version of a paper presented at the International Conference on Mechanical Engineering, Automation and Material Science (MEAMS2012), held 22-23 December 2012, Wuhan, China. Received 03 January 2013. Published as resubmitted by the authors 25 March 2013. 